\title{
Electrohydraulic incremental bulk metal forming
}

\author{
Lasse Langstädtler ${ }^{1,3^{*}}$, Holger Pegel ${ }^{1,3}$, Marius Herrmann ${ }^{1,3}$, Christian Schenck ${ }^{1,2,3}$, and Bernd Kuhfuss ${ }^{1,2,3}$ \\ ${ }^{1}$ Bremen Institute for Mechanical Engineering (bime), Badgasteiner Straße 1, 28359 Bremen, Germany \\ ${ }^{2}$ MAPEX - Center for Materials and Processing \\ ${ }^{3}$ University of Bremen
}

\begin{abstract}
Electrohydraulic forming is a working media based high speed technique that is usually applied for sheet metal processing. In this process a shock wave acts as a flexible punch that transmits the punching force in a very short period of time. This force is usually used to accelerate the workpiece towards the passive tool. In Contrast to sheet forming, the electrohydraulic method is still not adapted to bulk forming. Although, the exchange of a mechanical rigid punch by a shockwave with a flexible shape enables special advantages especially if parts with millimeter dimensions or smaller are to be processed. But in case of deep and small cavities with a high aspect ratio are to be filled, the forming energy is not transferable within one shock wave. To overcome these obstacles the incremental electrohydraulic forming is introduced. As an example, the electrohydraulic extrusion of cylindrical samples (aluminum A199.5) with an initial diameter of $1.5 \mathrm{~mm}$ was performed with a series of consecutive shock waves.
\end{abstract}

Keywords: Micro forming, Extrusion, Aluminum

\begin{tabular}{ll}
\hline Nomenclature \\
\hline$\alpha_{\mathrm{e}}$ & extrusion angle \\
$\mathrm{C}$ & capacitance \\
$\mathrm{d}_{\mathrm{b}}$ & bulge diameter \\
$\mathrm{d}_{\mathrm{d}}$ & guiding channel diameter \\
$\mathrm{d}_{\mathrm{e}}$ & extrusion channel diameter \\
$\mathrm{d}_{\mathrm{eS}}$ & extruded sample diameter \\
$\mathrm{d}_{\mathrm{s}}$ & initial sample diameter \\
$\mathrm{d}_{\mathrm{w}}$ & wire diameter \\
$\Delta \mathrm{e}$ & step size \\
$\mathrm{e}$ & extrusion height \\
$\mathrm{E}_{\mathrm{C}}$ & loading energy of the capacitor \\
$\mathrm{E}_{\mathrm{R}}$ & relative energy \\
$\mathrm{E}_{\mathrm{st}}$ & pulse energy \\
$\mathrm{E}_{\mathrm{V}}$ & vaporization energy \\
$\mathrm{EE}$ & total energy \\
$\mathrm{h}$ & bulge forming height \\
$\mathrm{h}_{\mathrm{R}}$ & relative bulge forming height \\
$\mathrm{I}$ & discharge current \\
$\mathrm{l}_{\mathrm{d}}$ & guiding channel length of the die \\
$\mathrm{l}_{\mathrm{e}}$ & extrusion channel length of the die \\
$\mathrm{l}_{\mathrm{S}}$ & initial sample length \\
$\mathrm{l}_{\mathrm{w}}$ & wire length \\
$\mathrm{s}_{0}$ & sheet metal thickness \\
$\mathrm{t}$ & time \\
$\mathrm{t}_{\mathrm{R}}$ & current rise time \\
$\mathrm{U}_{0}$ & loading voltage \\
\hline & \\
\hline
\end{tabular}

\section{Introduction}

To develop new metallic materials, time and cost intensive iterative procedures are required. Therefore, high-throughput technologies, as already used for medicine research, are suggested to decrease development time and reduce the costs [1]. Processes like single droplet solidification [2] provide rapidly generated and modified small material samples. Though, to characterize the new materials is still stated to be a fundamental problem [3].

One approach to characterize the material behavior of these micro-samples is to deform the samples and compare the required forces and the resulting strains to already characterized materials. Due to the low size and the spherical shape of the samples a suitable solution is a bulk forming operation. At conventional bulk forming of micro samples, the force transmission from punch to micro sample is an ambitious challenge. Especially, if the extrusion dies feature high aspect ratio between channel diameter and length. Here, as the mechanical punch also needs to provide the high aspect ratio, it tends to brake. In addition, close tolerances are required, as the samples material tends to flow into the gap between punch and die. Furthermore, when multiple bulk forming stages are aimed while pushing the sample completely through the die, the conventional punch is not flexible to undergo these stages within a single tool, Figure 1.

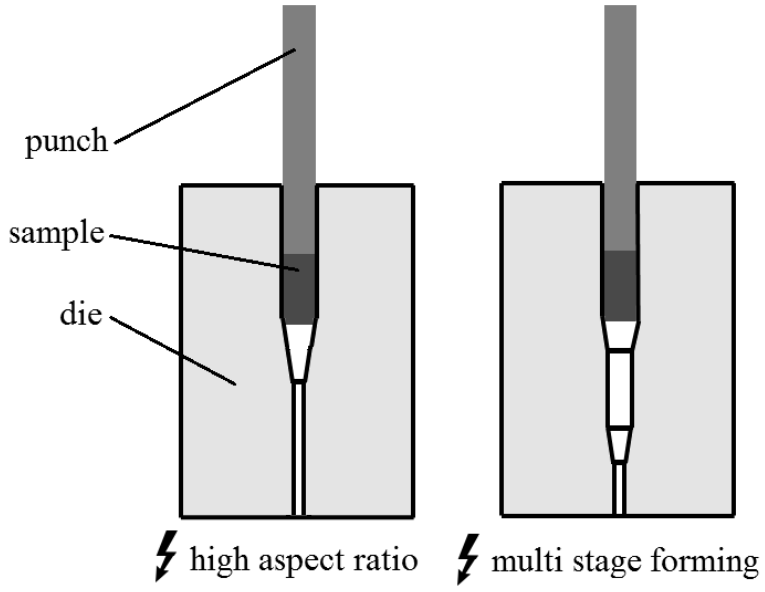

Fig. 1. Motivation for electrohydraulic bulk forming.

\footnotetext{
Corresponding author: langstaedtler@bime.de
} 
Consequently, a new bulk forming procedure is introduced as incremental electrohydraulic bulk forming. This high-speed process offers several advantages for micro forming like high deformation rates and a contactless force transmission $[4,5]$. Here the idea is to characterize the micro samples while they are incrementally formed into deep dies with different forming stages that cause defined forming-load conditions. The increments thereby should be quite small to sample the stress-strain curve with high resolution. Comparing measured deformations with simulation results enables the determination of characteristic values that describe the material behavior. In the process shock waves caused by an electric spark or a wire explosion in a working media filled pressure chamber are an approach to act as flexible punches. The energy $\mathrm{E}_{\mathrm{V}}$ is required to vaporize the wire and to build the shock wave. The electrohydraulic punch transmits the punching force in a few microseconds with high pressures up to several GPa [6].

By the action of force, the workpiece is pushed towards and in the passive tool, e.g. an extrusion die. In the die the workpiece material is deformed. Conventionally, the process energy is usually provided completely by a single shock wave. When deep and small cavities with a high aspect ratio are used for extrusion, multiple shock waves are required for an incremental extrusion, as the energy cannot be transferred within one single step. With the proposed new incremental method with flexible punch, extrusion in multi-stage dies including both compression and expansion operations are enabled.

In order to extrude micro samples by the action of shock waves, the energy transmission of the shock waves needs to be controlled. This was investigated by sheet metal bulge forming tests. Afterwards, by applying consecutive shock waves, the incremental bulk forming was investigated for the extrusion of micro samples. Here, the use of cylindrical samples should enable a repeatable testing due to its constant diameters as they vary for spherical microscopic samples [7]. The shock waves were varied in the amount of energy which was stepwise applied and the influence on the extrusion into the depth of the die was investigated.

\section{Experimental set-up}

The shock waves were generated by short circuiting an LC-resonator over a vaporizing aluminum wire. The wire had a diameter of $\mathrm{d}_{\mathrm{w}}=0.3 \mathrm{~mm}$ and a length of $1_{\mathrm{w}}=20 \mathrm{~mm}$ and was placed in a pressure chamber filled with distilled water. The water was renewed after each vaporization due to pollution of small aluminum particles that stay in the water after vaporization. The resonator had a capacitance of $\mathrm{C}=100 \mu \mathrm{F}$ and the loading voltage $\mathrm{U}_{0}$, and in consequence the loading energy $\mathrm{E}_{\mathrm{C}}=0.5 \cdot \mathrm{C} \cdot \mathrm{U}_{0}{ }^{2}$. The loading voltage was varied from $\mathrm{U}_{0}=0.5 \mathrm{kV}$ to $\mathrm{U}_{0}=2.7 \mathrm{kV}$ for sheet forming and from $\mathrm{U}_{0}=1.7 \mathrm{kV}$ to $\mathrm{U}_{0}=2.7 \mathrm{kV}$ for bulk forming.

\subsection{Electrohydraulic sheet metal bulging}

Sheet bulge forming (bulge diameter $\mathrm{d}_{b}=15 \mathrm{~mm}$, sheet thickness $\mathrm{s}_{0}=1 \mathrm{~mm}$, sheet dimension $50 \times 50 \mathrm{~mm}^{2}$, sheet material $\mathrm{AlMg} 3$ ) tests were performed to investigate the possibility to dose the shock wave, Figure 2 . The relative energy $E_{R}$, the quotient of the loading energy $E_{C}$ and the vaporization energy $E_{V}$, was varied from $E_{R}<1$ (partial vaporization) to $E_{R}>1$ (increased energy deposition), Equation 1.

$$
E_{R}=E_{C} / E_{V}
$$

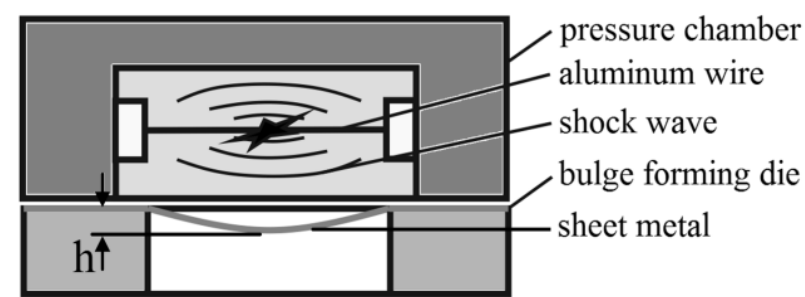

Fig. 2. Bulge forming set-up.

To vaporize an aluminum wire an amount of $14 \mathrm{MJ}$ per kilograms is required [8]. $\mathrm{E}_{\mathrm{V}}$ can be calculated theoretically. In this set-up, for three different wire diameters of $\mathrm{d}_{\mathrm{w}}=0.3 \mathrm{~mm}, \mathrm{~d}_{\mathrm{w}}=0.4 \mathrm{~mm}$ and $\mathrm{d}_{\mathrm{w}}=0.5 \mathrm{~mm}$, the vaporization energies are $\mathrm{E}_{\mathrm{V} 0.3} \approx 50 \mathrm{~J}$, $\mathrm{E}_{\mathrm{V} 0.4} \approx 100 \mathrm{~J}$ and $\mathrm{E}_{\mathrm{V} 0.5} \approx 150 \mathrm{~J}$. For each relative energy the characteristic discharge current $\mathrm{I}$ and the forming height $h$ were measured. The relative forming height $h_{R}$ was calculated with equation 2 .

$$
h_{R}=h\left(E_{R}\right) / h\left(E_{R}=1\right)
$$

\subsection{Electrohydraulic bulk forming}

Cylindrical aluminum (A199.5) samples with an initial diameter of $\mathrm{d}_{\mathrm{s}}=1.5 \mathrm{~mm}$ and an initial length of $1_{\mathrm{s}} \approx 3.0 \mathrm{~mm}$ were electrohydraulically extruded to a diameter of $\mathrm{d}_{\mathrm{e}}=1.28 \mathrm{~mm}$, Figure $3 \mathrm{a}$ ). Both end faces were initially flat. The length of the extrusion channel was $1_{e}=6 \mathrm{~mm}$. The extrusion die, made of unhardened St355 steel without reinforcement, had a diameter of $\mathrm{d}_{\mathrm{d}}=1.5 \mathrm{~mm}$ and a length of $\mathrm{l}_{\mathrm{d}}=5.2 \mathrm{~mm}$ in the first part of the die. The extrusion angle was $\alpha_{\mathrm{e}}=128^{\circ}$. The extrusion was performed stepwise by transferring different amounts of pulse energy $E_{\mathrm{st}}\left(\mathrm{E}_{\mathrm{st}}=162 \mathrm{~J}\right.$, $E_{s t}=288 \mathrm{~J}$ and $E_{s t}=382 \mathrm{~J}$ ) while keeping $E_{s t}$ constant for the consecutive shock waves of one extrusion. The total energy $\Sigma \mathrm{E}$ was calculated with Equation 3 and the current and voltage curve were measured, Figure $3 b$ ).

$$
\Sigma E=\Sigma E_{s t}
$$

The depth e was measured with a laser linetriangulation scanning set-up [5]. The sample was completely plunged in the extrusion channel of the die if $\mathrm{e}=5.2 \mathrm{~mm}$ was reached. The difference between each step was calculated as step size $\Delta \mathrm{e}$ with Equation 4.

$$
\Delta \mathrm{e}=\mathrm{e}_{\mathrm{i}+1}-\mathrm{e}_{\mathrm{i}}
$$


a)
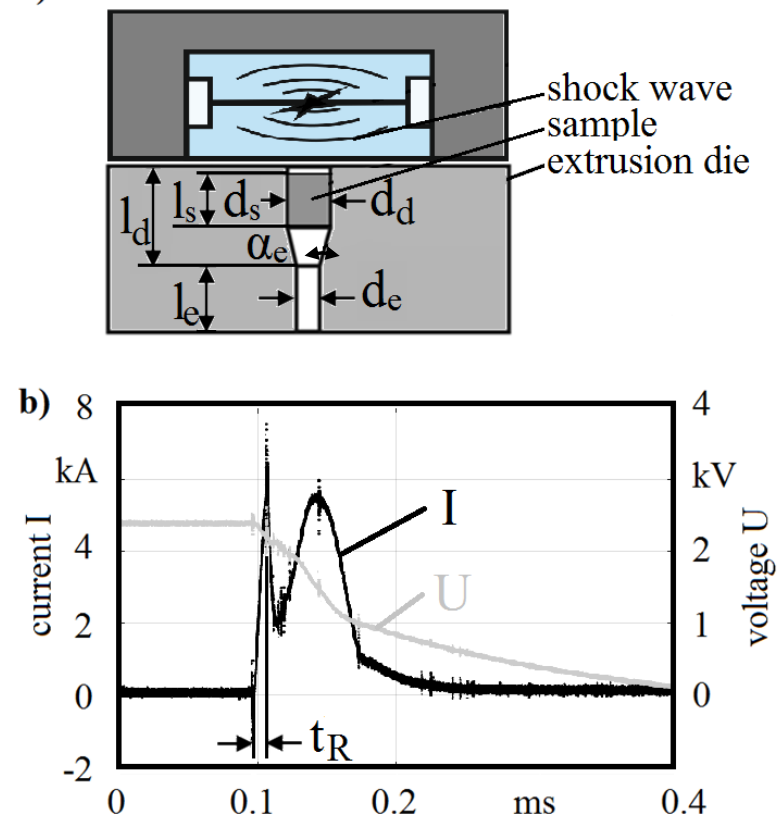

Fig. 3. Experimental set-up: a) pressure chamber with extrusion die, b) typical discharge current $\mathrm{I}$ and voltage $\mathrm{U}$ for $\mathrm{U}_{0}=2.4 \mathrm{kV}$.

\section{Experimental results}

\subsection{Electrohydraulic sheet metal bulging}

The relative forming height $h_{R}$ increased with the relative energy $E_{R}$, Figure $4 a$ ). A forming was even reached for $E_{R}<1$. Each $E_{R}$ resulted in a characteristic discharge current chart, Figure $4 b$ ). With $\mathrm{E}_{\mathrm{R}}<0.3$ the wire did not vaporize and the electrical circuit kept closed which resulted in an oscillation of the current without forming of the sheet metal. With $0.3<\mathrm{E}_{\mathrm{R}}<1$ the circuit broke by melting or vaporizing of the wire but the energy was not high enough to ignite a plasma channel. Hence, the capacitor was not completely discharged. However, the discharge resulted in a force that was high enough to deform the sheet metal. For $E_{R}>1$ the wire was vaporized completely and the adjunctive plasma kept the circuit closed until the capacitor was completely discharged. Further increasing $E_{R}$ decreased the peak rise time $t_{R}$ as the wire vaporized faster. Thus, by varying $E_{R}$, the shock wave was adjustable and the current charts qualitatively characterized the kind and strength of the discharged energy. The wire diameter $d_{w}$ did not influence the resulting relative forming heights $h_{R}$. Consequently, any chemical reactions of the vaporized aluminum did not contribute to the forming. However, the absolute forming steps decreased with decreasing $\mathrm{d}_{\mathrm{w}}$.
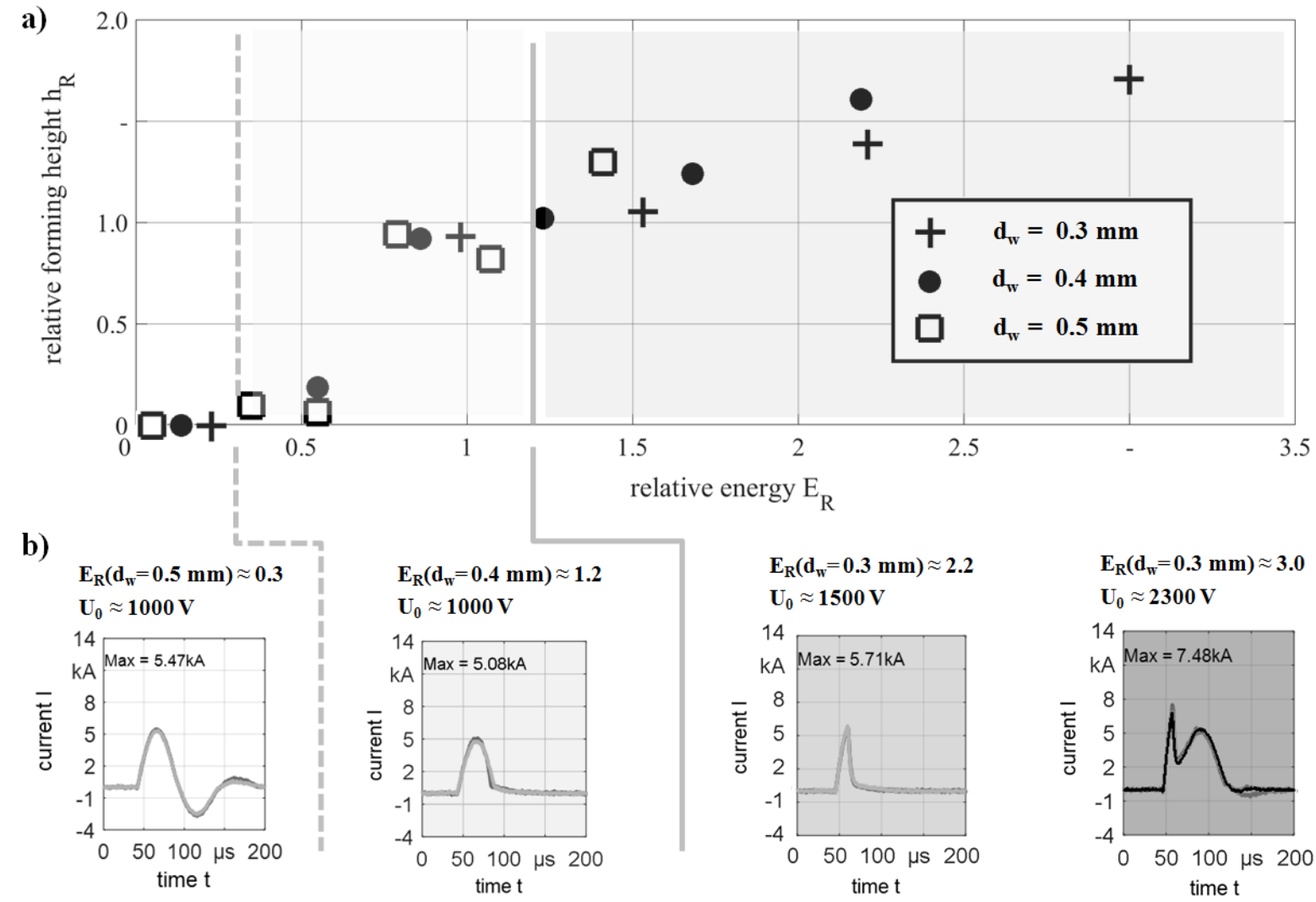

Fig. 4. Sheet metal bulging with different wire diameters $d_{w}$ : a) relative forming height $h_{R}$ as a function of the relative energy $\left.E_{R}, b\right)$ example of characteristic discharge currents I for different $E_{R}$. 


\subsection{Electrohydraulic bulk forming}

The extrusion started at $\mathrm{e} \approx 2.0 \mathrm{~mm}$ due to the length of the samples. Increasing $\Sigma \mathrm{E}$ resulted in an increase of the extrusion depth e, Figure 5. With the shown set-up it was possible to repeatedly generate shock waves that were gentle enough to incrementally extrude micro samples. For $\mathrm{E}_{\mathrm{st}}=162 \mathrm{~J}$ the extrusion stopped after a small deformation. However, a complete extrusion with $\mathrm{E}_{\mathrm{st}}=288 \mathrm{~J}$ and $\mathrm{E}_{\mathrm{st}}=382 \mathrm{~J}$ was possible.

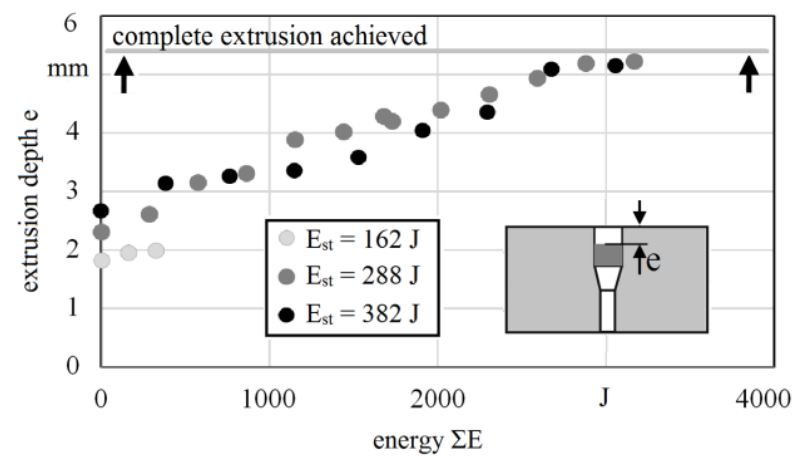

Fig. 5. Extrusion depth e for incremental extrusion.

Concerning the geometry of the samples, a convex deformation of the end face in the direction of extrusion (B) and an increase of the length of the sample was recognized, Figure 6. This is comparable to conventional extrusion. The other end face (A) in contrast exhibits a concave deformation. Thus, the hydraulic punch features a flexible shape. Both shapes of the end faces could be explained by friction between the sample and the die that reduced the axial material flow relatively to the center of the sample.

a)

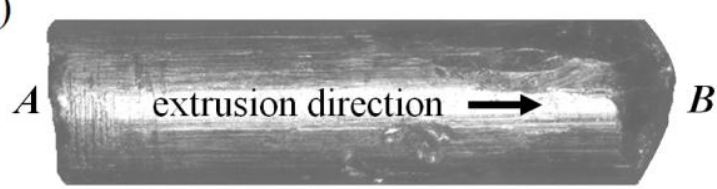

b)

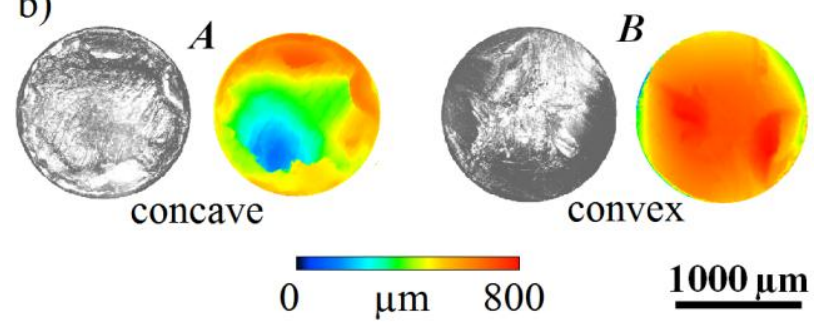

Fig. 6. Incrementally extruded A199.5 cylindrical sample $\left(E_{s t}=382 \mathrm{~J}\right)$ : a) side view, b) concave and convex face of the sample.

In Figure 7, the subsequent step size $\Delta \mathrm{e}$ is plotted as a function of the actual extrusion height e. The step size decreased with an increase of the extrusion depth. Though, until now it is not clear which effects are mainly to be addressed causing the spreading of the achieved results.

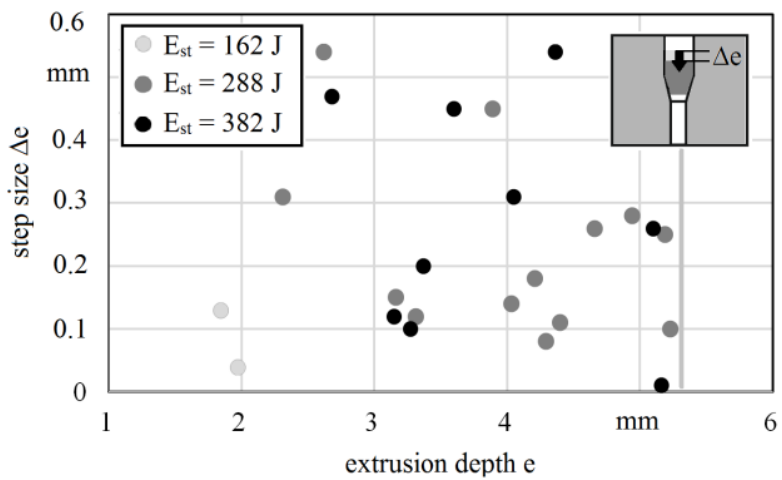

Fig. 7. Step size $\Delta \mathrm{e}$ as function of the actual extrusion depth e.

Comparing both, the diameter of the extrusion channel of the die $\mathrm{d}_{\mathrm{e}} \approx 1.28 \mathrm{~mm}$ and the diameter of the extruded sample $\mathrm{d}_{\mathrm{es}} \approx 1.27 \mathrm{~mm}$, a very good fit was reached for both energies, although the extrusion die was neither hardened nor reinforced. There was no need of pre-strains, which are conventionally required for extrusion dies, to compensate the elastic spring back of the sample after extrusion. In contrast to conventional extrusion operations, the samples diameter was nearly the same (even lower) than the dies diameter $d_{e s}<d_{e}$ and in addition, this was reached with a very simple die which had a low stiffness. This can be explained by the very high deformation rate that results in an increased samples material plasticity.

As the die as well is loaded by the shock wave, it possibly tends to deform. Hence, a further explanation for this good replication is an elastic deformation of the die which can lead to a reduction of the diameter which operates like a pre-strain. This is underlined by the fact, that different energies had the same influence on the increase of extrusion depth which indicates a selfregulation feature of the process.

\section{Conclusion}

In this contribution the potential of electrohydraulic forming was analyzed to fully extrude micro samples within deep dies with the purpose to establish a new method for high throughput materials testing. Therefore, two fundamental questions had to be answered: i) How and in which range can the shock wave that result in an action of force be dosed to realize an incremental forming, and ii) can the shock wave be used as a flexible punch in bulk metal forming, especially extrusion? The first question was examined with sheet metal bulge testing with the following conclusions:

- The action of force could be controlled by the relative energy $E_{R}$ that is the proportion of supplied energy and vaporization energy of the wire which itself is a function of the wire diameter $\mathrm{d}_{\mathrm{w}}$.

- With values of $E_{R}<1$ small forming was achievable although the wire did not completely vaporize or melt - this enabled very small deformation steps. 
- The relative energy could be monitored by the discharge current whereat the kind of force generation was imprinted in the current curve progression.

The second question was analyzed with electrohydraulic extrusion experiments with the following results:

- Bulk metal micro-forming and especially full extrusion was possible with the means of electrohydraulic forming.

- Even full extrusion within a deep cavity -with an aspect ratio of 5-could be realized.

- Sufficient small forming increments with a step size of $100 \mu \mathrm{m}$ and below were attained.

- The shock wave easily adapted to changing shapes of the workpiece end face.

- Due to high pressures for bulk forming according to the investigated step sizes extrusion was realized with relative energies of $E_{R}>5$.

These results state the principle feasibility of the proposed high throughput materials testing approach. Additionally, three aspects inherently follow from these findings and will be subject of future work:

- Multistage full extrusion in one single die that is out of question with a mechanical punch will be enabled with the electrohydraulic technique.

- Even full lateral and backward extrusion should be feasible by using a shock wave instead of a mechanical punch.

- The tool design and manufacturing will be strongly simplified if the special demands of high speed forming are taken into account. Hardening and reinforcing of the tools may not be mandatory as shown by the extrusion experiments.

\section{Acknowledgments}

The authors would like to gratefully acknowledge the financial support of subproject D04 within the Collaborative Research Center SFB 1232 "Farbige Zustände" by the German Research Foundation (DFG).

\section{References}

1. L. Mädler, 4th International conference on Nanomanufacturing Bremen (2014)

2. N. Ellendt, N. Ciftci, C. Goodreau, V. Uhlenwinkel, L. Mädler, IOP Conference Series: Materials Science and Engineering 117 (1) (2016)

3. A. Beinhauer, C. Heinzel, O. Riemer, Fachbeiträge 8. Kolloquium Mikroproduktion, Editor: F. Vollertsen, C. Hopmann, V. Schulze, J. Wulfsberg, BIAS Verlag (2017)
4. L. Langstädtler, L. Schönemann, C. Schenck, B. Kuhfuss, ASME Journal of Micro- and Nano-Manufacturing, Volume 4, Issue 2 (2016)

5. L. Langstädtler, M. Herrmann, C. Schenck, B. Kuhfuss, 8th Conference on Tribology in Manufacturing Processes \& Joining by Plastic Deformation, Denmark (2018)

6. S.F. Golovashchenko, Project report - Electrohydraulic Forming of Near-Net Shape Automotive Panels (2013)

7. L. Langstädtler, H. Pegel, M. Herrmann, C. Schenck, D. Stöbener, J.F. Westerkamp, A. Fischer, B. Kuhfuss, International Conference on High Speed Forming, Columbus - Ohio, (2018)

8. M. Löffler, H.A. Wieland, International Conference on Pulse Power Applications, E.16/1-7, (2001) 